\title{
INVESTIGATION OF FILTERING AND OBJECTS DETECTION ALGORITHMS FOR A MULTIZONE IMAGE SEQUENCE
}

\author{
N. A. Andriyanov ${ }^{1,2 *}$, K.K. Vasil'ev ${ }^{1}$, V.E. Dement'ev ${ }^{1}$ \\ ${ }^{1}$ Ulyanovsk State Technical University, 432027 ul. Severny Venets, h. 32, Ulyanovsk, Russia - nikita-and-nov@mail.ru \\ ${ }^{2}$ Ulyanovsk Institute of Civil Aviation, 432071 ul. Mozhaiskogo, h. 8/8, Ulyanovsk, Russia
}

\section{Commision II, WG II/5}

KEY WORDS: Filtering, Multizone Images, Random Fields, Doubly Stochastic Models, Model with Multiple Roots, Anomalies Detection

\begin{abstract}
:
The problem of detecting objects on a sequence of images with a complex structure is considered. Optimal and quasi-optimal algorithms for processing multidimensional images have been synthesized and investigated. Improved detection efficiency has been obtained by adequately describing real data using doubly stochastic random fields. The possibility of describing Earth remote sensing data using doubly stochastic models is investigated. The possibility of obtaining significant gains when filtering satellite material and detecting extended objects on it due to the adaptive structure of such models and processing time sequence of multizone images as a single multidimensional dataset is shown. The gains for filtering algorithms in the error variance are about $80 \%$ comparing single frame processing, and the gains for detecting algorithms in the signal/noise ratio are about $70 \%$ comparing single frame processing.
\end{abstract}

\section{INTRODUCTION}

One of the key features of real satellite imagery is their pronounced spatial heterogeneity. It is associated with the variety of forms and textures of various objects observed from space. Attempting to describe the entire image using wellknown homogeneous models (Gonzalez, 2006), e.g., Gibbs, autoregressive, wave, and others, leads to significant errors. In papers (Vasiliev, 2017b,c, Andriyanov 2017,2018, Vasiliev, 2018) it was proposed to use doubly stochastic models (DSM). These models not only allow one's to generate images with varying probabilistic properties, but are also quite simple for analysis and used to form various processing algorithms on its basis. In this paper, we consider the use of DSM during filtering algorithms for time sequences of spatially inhomogeneous multizone images (MZI) and the detection of objects against their background.

\section{IMAGES SEQUENCE FILTERING}

First, our task is filtering sequence consisting of a set of twodimensional frames. So, the images are implementations of a discrete time RF, given on a multidimensional grid (Vasiliev, 2016,2017a) $J_{t}=\left\{\bar{j}=\left(j_{1}, j_{2}, j_{3}\right) ; j_{l}=1, M_{l}, l=1,2,3\right\}$, where $j_{1}, j_{2}$ are space coordinates, $j_{3}$ is the frame number. Then the first frame of the MZI is determined by twodimensional $\operatorname{RF}\left\{x_{i j}^{1}\right\}$, given on the grid $\left\{(i, j) ; i=1, M_{1} ; j=1, M_{2}\right\}$. We can write $\left\{x_{i j}^{1}\right\}$ using DSM

$$
x_{i, j}=A+a_{i, j}+F_{A R M R\left(k_{1}, k_{2}\right)}\left(P_{1}+\rho_{1 i, j}, P_{2}+\rho_{2 i, j},\left(B+b_{i, j}\right) \beta_{i, j} \xi_{i, j}\right)
$$

where $A, P_{1}, P_{2}, B$ are the expected values of model parameters; $F_{A R M R\left(k_{1}, k_{2}\right)}\left(\rho_{1}, \rho_{2}, b\right)$ is transformation describing an autoregression model with multiple roots [2] (ARMR) $\left(1-\rho_{1} z_{1}^{-1}\right)^{k_{1}}\left(1-\rho_{2} z_{2}^{-1}\right)^{k_{2}} x_{i, j}=b \beta \xi_{i, j}, \quad$ where $K_{1}, K_{2}$ are the multiplicities of the model; $\beta$ is normalization factor; $z_{k}^{-1}$ is shift $\quad z_{1}^{-l_{1}} z_{2}^{-l_{2}} x_{i, j}=x_{i-l_{1}, j-l_{2}}$; $a_{i j}=F_{A R M R\left(K_{a 1}, K_{a 2}\right)}\left(r_{a 1}, r_{a 2}, \gamma_{a}, \xi_{a i j}\right), \rho_{1 i j}=F_{A R M R\left(K_{1 \rho 1}, K_{2 \rho 1}\right)}\left(r_{11}, r_{12}, \gamma_{1}, \xi_{1 j j}\right)$, $\rho_{2 i j}=F_{A R M R\left(K_{1 \rho 2}, K_{2 \rho 2}\right)}\left(r_{21}, r_{22}, \gamma_{2}, \xi_{2 i j}\right), \quad b_{i j}=F_{A R M R\left(K_{D_{1}}, K_{D_{2}}\right)}\left(r_{\beta 1}, r_{\beta 2}, \gamma_{\beta}, \xi_{\beta i j}\right)$ are random fields of parameters; $\xi_{i j}, \xi_{a i j}, \xi_{1 i j}, \xi_{2 i j}, \xi_{\beta i j}$ are Gaussian white noise; $i=1, \ldots, M_{1}, j=1, \ldots, M_{2}$.

Subsequent frames are obtained by $\operatorname{RF}\left\{x^{k}{ }_{i j}\right\}, k=2, \ldots, N$, on the grid $J_{t}$. Let the matrix of interframe correlations $R$ be determined as a result of long-term observations. Then to simulate the second and subsequent frames, one's can use the following relationship

$$
x_{i, j}^{k}=\sum_{l=1}^{k} R(l, k) x_{i, j}^{1}+\sum_{i_{1}=1}^{M_{1}} \sum_{j_{1}=1}^{M_{2}} v_{i_{1}, j_{1}} \xi_{i_{1}, j_{1}}^{k}
$$

where $v_{i_{1}, j_{1}}$ are elements of a triangular matrix $V$, for which $V V^{T}=B ; \quad B$ is covariance matrix of the first frame. Note that the described approach to simulating a sequence of twodimensional frames can be used for imitation of the MZI time sequence.

Earlier (Vasiliev, 2017b,c), it was shown that this model can be represented as the following vector stochastic equation

\footnotetext{
* Corresponding author
} 


$$
\bar{x}_{i, j}=\varphi_{i, j}\left(\bar{x}_{i, j}\right)+\bar{\xi}_{i, j}
$$

where $\bar{\xi}_{i, j}$ is the vector of additive white Gaussian noise.

Then, to filter such a three-dimensional RF, one's can use a doubly stochastic filter (DSF), given that at each point with coordinates $(i, j)$ we have not only a single scalar observation $z_{i, j}$, but the vector of observations.

Figure 1 shows the efficiency of doubly stochastic filtering. Figure 1a (X-axis is number of frames in Multizone Image, Yaxes is Filtering Error Dispersion) corresponds to the dependence of the variance of filtering errors on the number of frames used, the dotted line shows the error for single frame. Figure $1 \mathrm{~b}$ (X-axis show interframe correlation coefficient in Multizone Image, Y-axes show Filtering Error Dispersion) illustrates the dependence of the variance of filtering errors on the interframe correlation for 2, 4, and 10 frames, respectively.

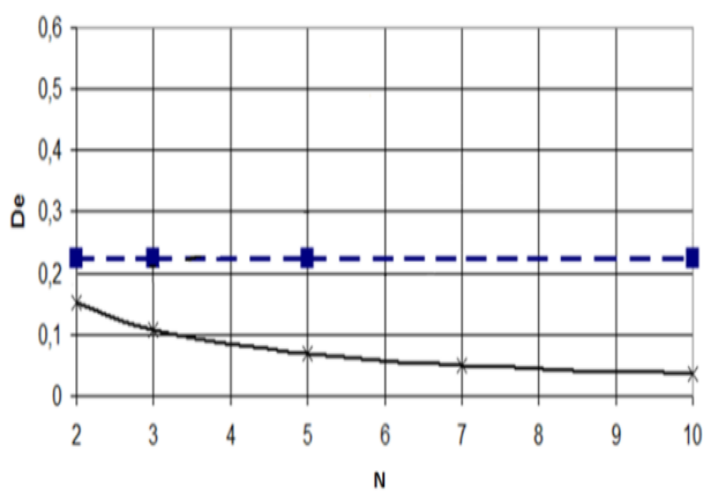

a

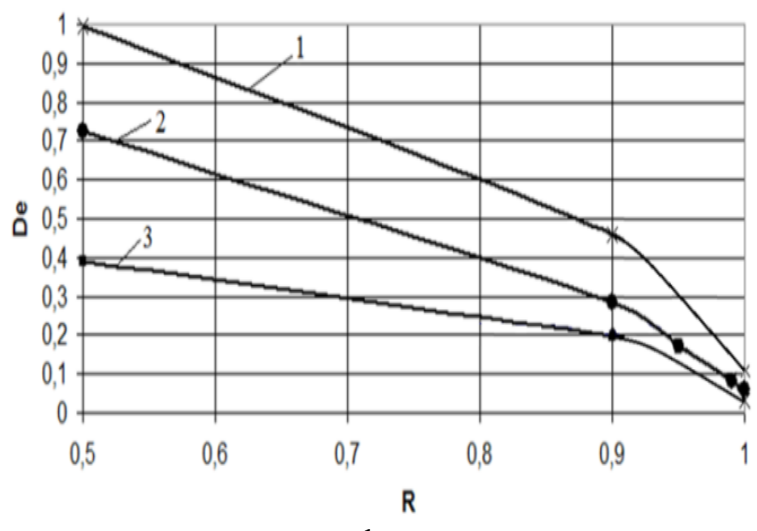

b

Figure 1. Multizone Image Filtering Efficiency

The analysis shows that the variance of the filtering error tends to the minimum values, if the number of frames is about 10 . At the same time, if the frames are weakly interconnected, then increasing the number of frames significantly increases the filtering efficiency, but such filtering is not acceptable. And with $R$ tending to 1 , we get relatively small variances of errors.

Figure 2 shows an example of filtering an artificially noisy fragment of a real satellite image obtained from the Landsat 8 spacecraft.

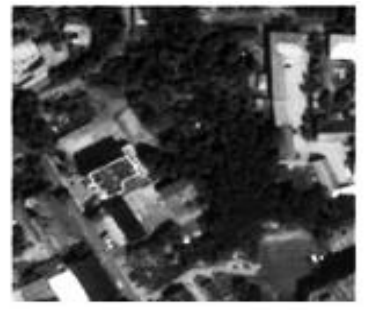

a

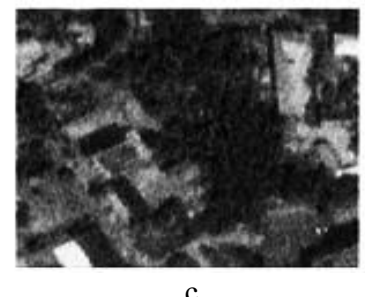

c

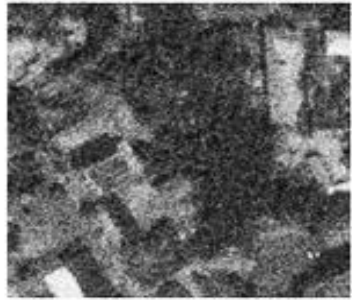

b

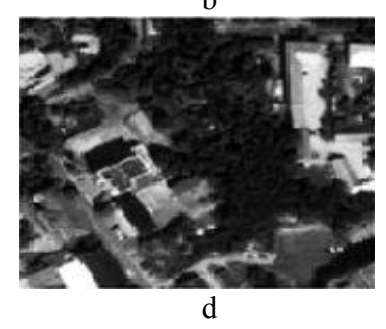

Figure 2. Filtering satellite images: source image (a), noisy image (b), one frame filtering (c), eight frame filtering (d).

Visual analysis of figure 2 shows that an increase in the number of image frames leads to a significant increase in filtering efficiency. In particular, for the present case, the gain in the variance of the filtering error is about $80 \%$ when using all eight available spectral zones as compared with a single twodimensional frame.

Figure 3 (X-axis is Noise Variance, Y-axes is Filtering Error Dispersion) shows the dependences of the image filtering error variance on the noise variance: A1 is a Kalman vector filter without interpolation; A2 is a discrete Wiener filter, A3 is a Kalman filter with interpolation, A4 is a DSF (Vasiliev, 2018).

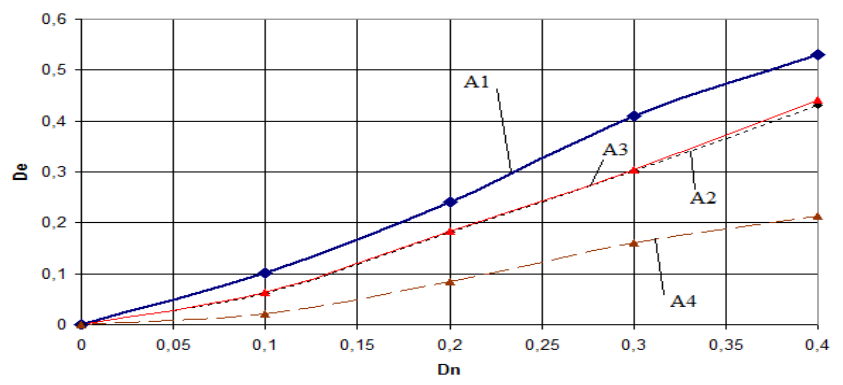

Figure 3. Filtration efficiency during satellite images processing

A simple analysis of the curves in Figure 3 shows the effectiveness of the proposed filtering method, the gain of which compared to the other filters is up to $120 \%$ in terms of the variance of the estimation error. Moreover, there is an even stronger result. The DSF on average wins up to $15-20 \%$ in terms of the variance of the estimation error in filters A1-A3, even if the latter are already applied to an image segmented by humans. This is due to the possibility of adaptive adjustment of the parameters of the DSF under the smoothly varying probability and correlation parameters of the image.

\section{OBJECTS DETECTION}

Usually (Gonzalez, 2006, Vasiliev 2016,2017a), the problem associated with the detection of objects is represented as the problem of distinguishing between two hypotheses about the presence or absence of an anomaly in a certain area of the image. The observation model, which is most often used when detecting, is an additive mixture of correlated background, white noise, and samples of the useful signal. It was shown in 
(Vasiliev, 2016) that if such a model of observations is valid and the conditional distribution of observations with or without a useful signal can be approximated by Gaussian, then the decisive rule of the optimal detector against the background of a multidimensional random field takes the form

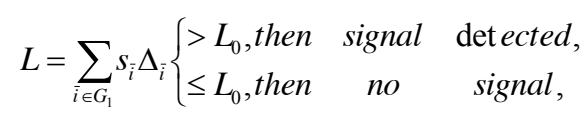

where $S_{\bar{i}}$ is value of the useful signal at a point with coordinates $\bar{i}=\left(i_{1}, i_{2}, \ldots, i_{N}\right) ; \Delta_{\bar{i}}=\sum_{\bar{j} \in G_{1}} V_{\bar{\theta} \bar{j}}^{-1}\left(z_{\bar{j}}-\hat{x}_{\bar{j}}\right) \quad$ is filtering error at a point $\bar{i}=\left(i_{1}, i_{2}, \ldots, i_{N}\right)$, normalized by noise variance $\theta_{\bar{i}} ; G_{1}$ is the area for which the signal presence hypothesis is being tested; $N$ is the dimension of RF; $L_{0}$ is threshold defined for a given false alarm probability.

We assume that it is necessary to test the hypothesis of the presence of a useful object at the last image in the time sequence of the MZI, provided that this object is not present at all other MZI in the sequence. Then the model of observations in the presence of a useful signal will be written in the form

$$
\begin{aligned}
& z_{i, j}^{k, T}=x_{i, j}^{k, T}+s_{i, j}^{k, T}+\theta_{i, j}^{k, T}, k=1,2, \ldots, N,(i, j) \in G_{0}^{k, T}, \\
& z_{i, j}^{k, T}=x_{i, j}^{k, T}+\theta_{i, j}^{k, T}, k=1,2, \ldots, N,(i, j) \notin G_{0}^{k, T}, \\
& z_{i, j}^{k, t}=x_{i, j}^{k, t}+\theta_{i, j}^{k, t}, k=1,2, \ldots, N, t=1, \ldots, T-1,
\end{aligned}
$$

where $G_{0}^{k, T}$ is region on the $k$-th frame of the MZI at time $T$; $s_{i, j}^{k, T}$ are the known samples of the useful object; $T$ is the moment of registration of the last image in the MZI sequence.

In the absence of a useful object, the observation model will be rewritten as a simple group of equalities

$$
z_{i, j}^{k, T}=x_{i, j}^{k, T}+\theta_{i, j}^{k, T}, k=1,2, \ldots, N, t=1,2, \ldots, T .
$$

Let use the Neyman-Pearson criterion (Scott 2018, Rahaman 2018), according to which the detection rule is chosen that provides the minimum value of the signal skip probability (the maximum probability of correct detection), provided that the probability of a false alarm does not exceed the specified value $P_{f}$. Thus, the optimal, in the sense of the Neyman-Pearson criterion, the detection rule maximizes

$$
P_{D}=1-P_{M}=\int_{G_{1}} \int \omega\left(\bar{z} / H_{1}\right) d \bar{z},
$$

where $\omega\left(\bar{z} / H_{1}\right) d \bar{z}$ is conditional posterior density of the probability distribution under the hypothesis of the presence of a signal.

Also it is necessary to introduce additional restriction

$$
\int_{\bar{G}_{1}} \int \omega\left(\bar{z} / H_{0}\right) d \bar{z}=P_{F}
$$

Let us calculate the characteristics of the detection of a point signal of level $S$, which may appear at time $i$ of discrete time. Decision rule is the following

$$
\lambda=S^{T}\left(P_{\ni}+V_{n}\right)^{-1}\left(\bar{z}_{S}-\hat{\bar{x}}_{\ni}\right)\left\{\begin{array}{l}
>\lambda_{0}-\text { signal }, \\
\leq \lambda_{0}-n o \text { signal } .
\end{array}\right.
$$

And finally we can rewrite (9) as in point-detection case

$$
\lambda=\frac{S\left(z_{S}-\hat{x}_{\ni}\right)}{V_{n}}\left\{\begin{array}{l}
>\lambda_{0}-\text { signal }, \\
\leq \lambda_{0}-\text { no } \text { signal } .
\end{array}\right.
$$

In the next step, we find the mathematical expectations and variances of the left side of the detector under the conditions of the presence and absence of a signal

$$
\begin{gathered}
M\left\{\lambda / H_{1}\right\}=\frac{S^{2}}{V_{n}}, M\left\{\lambda / H_{1}\right\}=\frac{S^{2}}{V_{n}}, \\
\sigma_{\lambda}^{2}=D\left\{\lambda / H_{1}\right\}=D\left\{\lambda / H_{0}\right\}=\frac{S^{2}}{V_{n}^{2}}\left(V_{n}+V_{e}^{2}\right) .
\end{gathered}
$$

Then the probability of a false alarm and the missing target can be written as follows

$$
\begin{gathered}
P_{F}=0.5-\Phi_{0}\left(\frac{\lambda_{0}}{\sigma_{\lambda}}\right) \text { - false alarm probability, } \\
P_{M}=0.5-\Phi_{0}\left(\frac{h-\lambda_{0}}{\sigma_{\lambda}}\right) \text { - signal skip probability, }
\end{gathered}
$$

where $\Phi_{0}$ is Laplace function, $h=S^{2} / V_{n}$.

On the basis of the presented observation model, an algorithm for detecting anomalies can be proposed, involving sequential filtering of individual MZI using the algorithm described above, calculating statistics (4) and comparing the result with a threshold value. Figure 4 (X-axis is Signal-to-Noise Ratio, Yaxes is Correct Detection Probability) shows the dependence of the probability of correct detection on the signal-to-noise ratio $q$ with a different number of frames in MZI. False alarm probability is $P_{f}=0.001$.

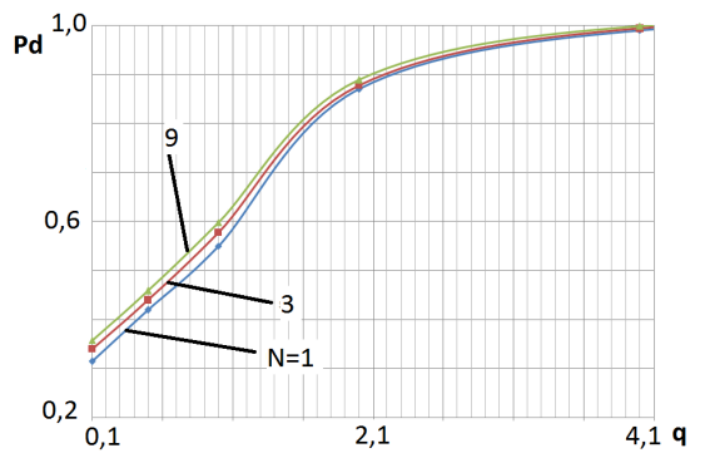

Figure 4. Detection efficiency for a sequence of images with different number of frames 
An analysis of the presented curves shows that for small signalto-noise ratios $(q<1)$, using a larger amount of MZI in a time sequence (up to 9 in the experiment) results in a $50-70 \%$ gain in signal-to-noise ratio.

The developed algorithm provides better results also in comparison with usual autoregression (Akashi 2016, Baragona 2015). Figure 5 (X-axis is Signal-to-Noise Relation, Y-axes is Correct Detection Probability) shows graphs of the probability of correct detection as a function of the signal-to-noise ratio. The solid line is the theoretical probability for the doubly stochastic model, the dashed line is the practical probability for the algorithm based on the doubly stochastic model, and the dash-dotted one for the algorithm based on the usual autoregression model.

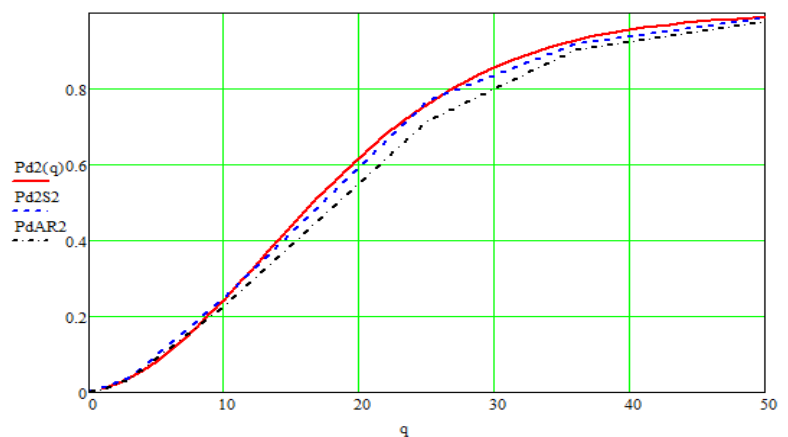

Figure 5. Probability of correct detection

It can be seen that detection based on the doubly stochastic model is more effective than detection based on the autoregression model. The gains at level 0,5 is about $20 \%$.

\section{CONCLUSION}

The possibility of describing Earth remote sensing data using DSM was investigated. The possibility of obtaining significant gains when filtering satellite material and detecting extended objects on it due to the adaptive structure of such models and processing time sequence of MZI as a single multidimensional population is shown. The gain of using sequence of images is obtained about $80 \%$ for filtering in error variance and $70 \%$ for detection in signal-to-noise ratio.

\section{ACKNOWLEDGEMENTS}

The study was supported by RFBR, project № 18-31-00056.

\section{REFERENCES}

R. Gonzalez, R. Woods 2006. Digital Image Processing. M.: Technosphere, $812 \mathrm{p}$.

Vasiliev K.K., Krasheninnikov V.R. 2017a. Statistical analysis of image sequences. - M .: Radio Engineering, 248 p.

Vasiliev K.K. 2016 Optimal signal processing in discrete time: a tutorial. - M .: Radio Engineering, 2016. - 288 p..

Vasiliev K. K., Andriyanov N. A. 2017b. Synthesis and analysis of doubly stochastic models of images. CEUR Workshop

Proceedings, Volume 2005, 2017, p. 145-154
Andriyanov N. A., Dement'ev V. E. 2018. Application of mixed models of random fields for the segmentation of satellite images. CEUR Workshop Proceedings, Volume 2210, 2018, p. 219-226.

Andriyanov N.A., Vasiliev K.K., Dementiev V.E. 2017 Anomalies detection on spatially inhomogeneous polyzonal images. CEUR Workshop Proceedings, Volume 1901, 2017, p. $10-15$

Vasiliev K. K., Dementiev V. E., Andriyanov N. A. 2017c. Filtration and restoration of satellite images using doubly stochastic random fields. CEUR Workshop Proceedings, Volume 1814, 2017, P. 10-20

K. Vasiliev, V. Dementiev and N. Andriyanov 2018. Representation and processing of multispectral satellite images and sequences. Procedia Computer Science 126 (2018), p. 4958.

https:// doi.org/10.1016/j.procs.2018.07.208

Clayton Scott 2018.

A Generalized Neyman-Pearson Criterion for Optimal Domain Adaptation. Proceedings of Machine Learning Research, vol 98: p.1-24, 2019

M. F. Rahaman and M. Z. Ali Khan 2018

Low-Complexity Optimal Hard Decision Fusion Under the Neyman-Pearson Criterion, IEEE Signal Processing Letters, vol. 25,2018 , p. $353-357$

F. Akashi and H. Dette 2016

Change point detection in autoregressive models with no moment assumptions. arXiv:1612.01520v1 [math.ST] 4 Dec 2016

R. Baragona, F. Battaglia and D. Cucina 2015

Empirical Likelihood for Outlier Detection and Estimation in Autoregressive Time Series https://doi.org/10.1111/jtsa.12145 